\title{
Intentional endoscopic nasopancreatic drainage to a pancreatic fistula in the treatment of disconnected pancreatic duct syndrome
}

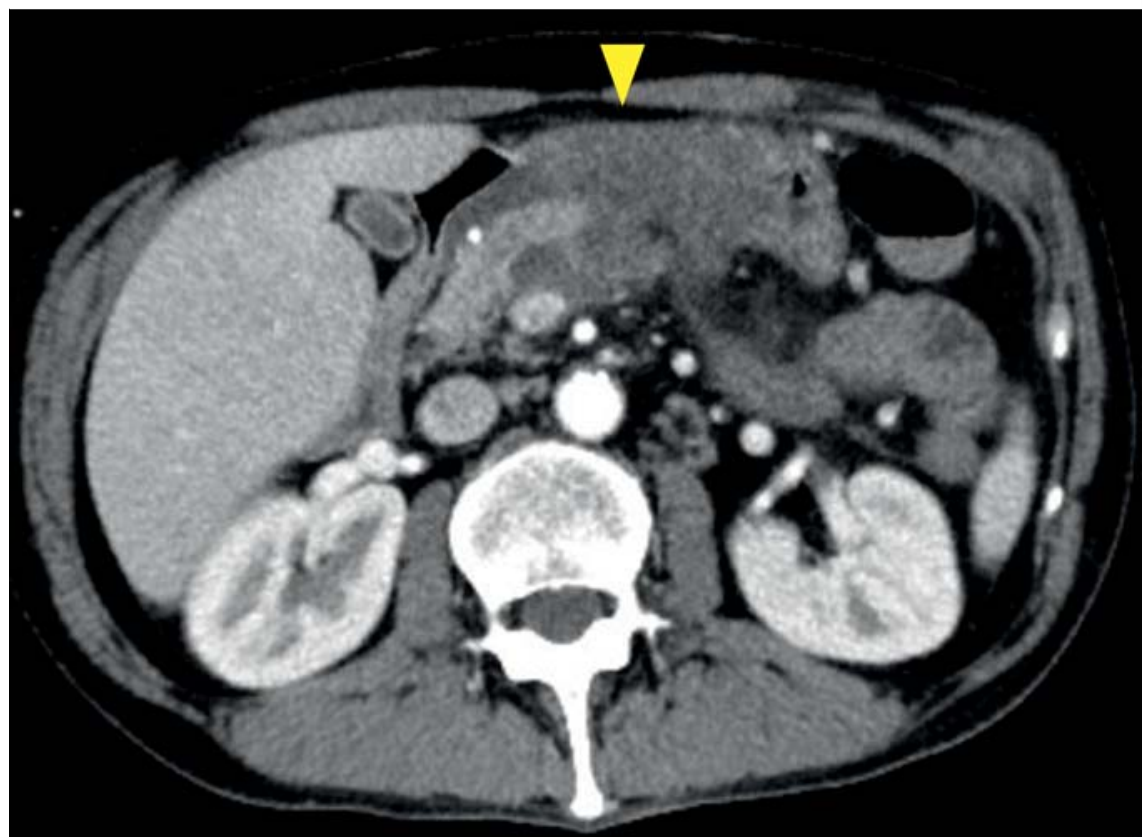

- Fig. 1 A 48-year-old man was diagnosed with disconnected pancreatic duct syndrome caused by severe acute pancreatitis. The pancreatic fistula was not encapsulated (arrowhead).

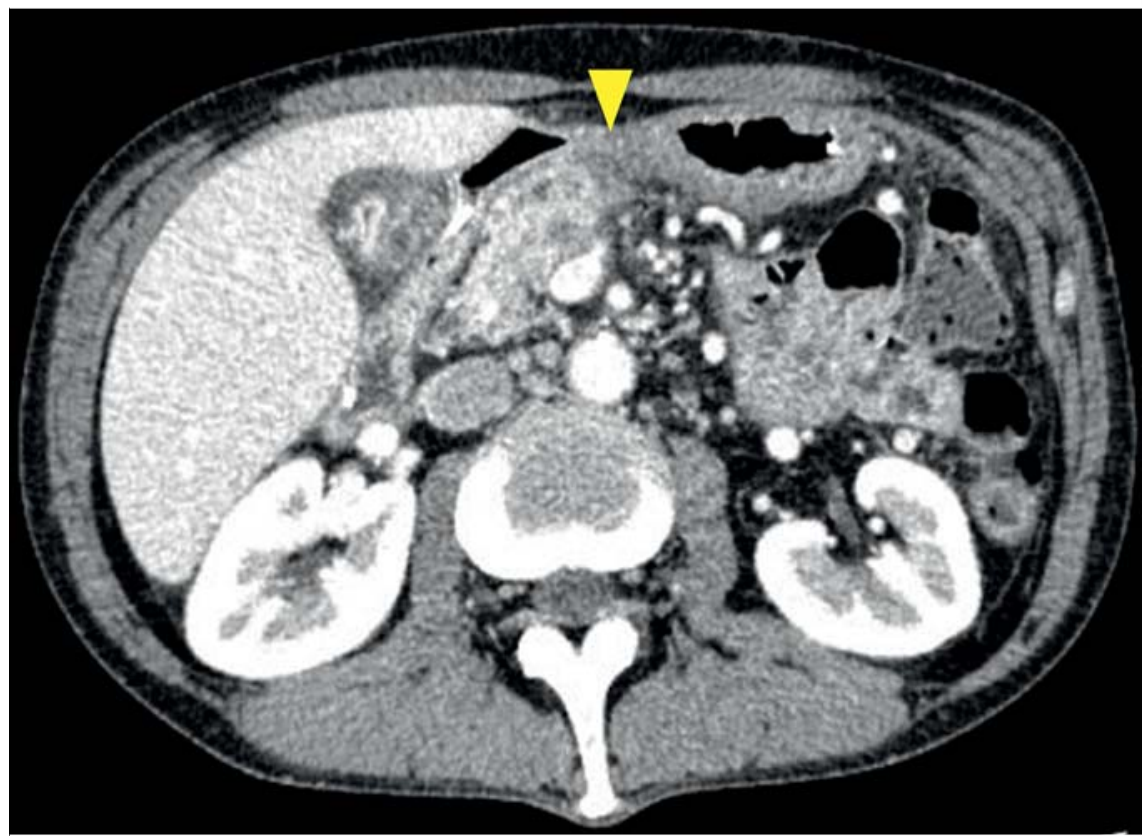

- Fig. 3 After waste fluid was controlled using endoscopic nasopancreatic drainage (ENPD), there was no peripancreatic fluid collection (arrowhead).

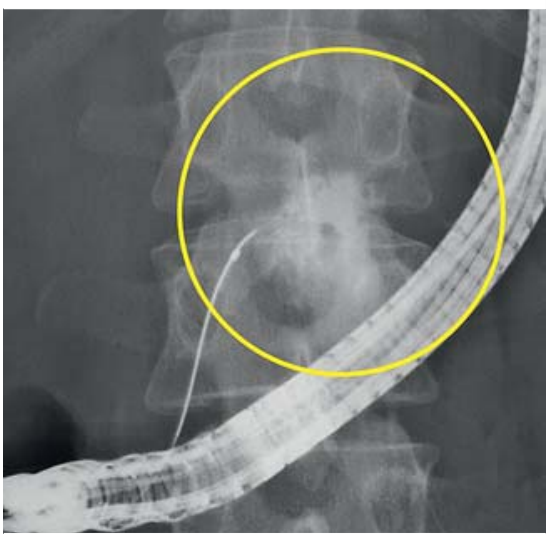

Fig. 2 Endoscopic retrograde pancreatography (ERP) showed that the main pancreatic duct (MPD) in the body of the pancreas was completely disrupted (circle).

Endoscopic transpapillary stenting and/ or transluminal drainage is recommended for disconnected pancreatic duct syndrome (DPDS). Transpapillary stenting can be considered, preferably with the stent bridging the disruption in the main pancreatic duct (MPD) [1,2]. Such treatment is currently feasible for patients with partial, but not complete, MPD disruption $[3,4]$. If this treatment fails, surgery is an alternative in patients with either partial or complete disruption - but the majority of these patients go on to develop diabetes mellitus [5]. For this reason, if possible, endoscopic treatment is preferred in order to preserve pancreatic function. We report a successful stent bridging technique for DPDS with complete MPD disruption after intentional endoscopic insertion of a nasopancreatic drainage (ENPD) tube into a pancreatic fistula.

A 48-year-old man was diagnosed with DPDS caused by severe acute pancreatitis. The patient suffered from recurring unbearable pain related to a pancreatic fistula. The pancreatic fistula was not encapsulated ( $\triangleright$ Fig.1), so we performed 


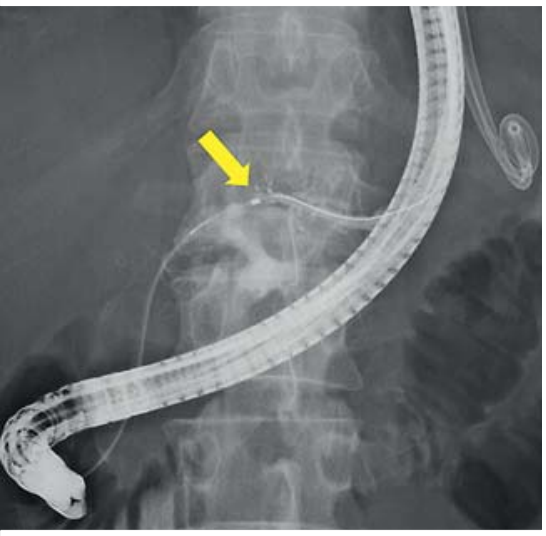

- Fig.4 Once the waste fluid was under control, we repeated ERP and identified the MPD in the tail of the pancreas beyond the disruption (arrow), which we successfully bridged.

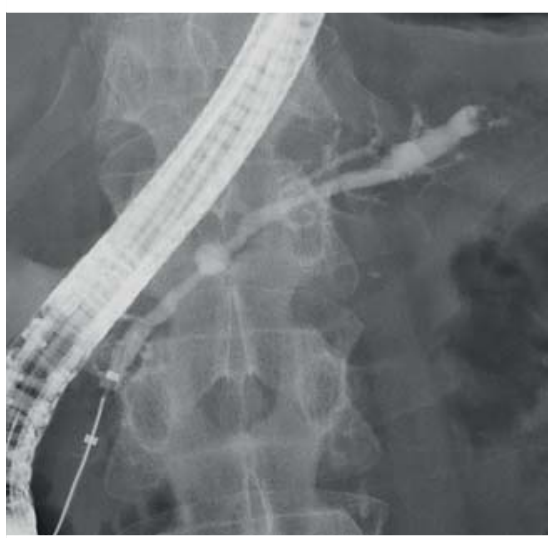

Fig. 5 Six months after the first treatment, we confirmed the absence of leakage under contrast pancreatography.

transpapillary stenting rather than transluminal drainage. According to endoscopic retrograde pancreatography (ERP), the MPD in the body of the pancreas was completely disrupted ( $\triangleright$ Fig. 2). The upstream MPD could not be visualized, and because it was difficult to bridge the disrupted MPD, we planned to temporarily place an ENPD tube in the unencapsulated pancreatic fistula. Flushing via an ENPD enabled cloudy waste fluid to be controlled, and peripancreatic fluid collection was absent ( $\mathbf{F i g . 3}$ ). Thereafter, we repeated ERP and identified the MPD in the tail of the pancreas beyond the disruption, which we successfully bridged (> Fig.4; $>$ Video 1 ). We then exchanged external for internal drainage and replaced the drain every 8-12 weeks. Six months later, we con-
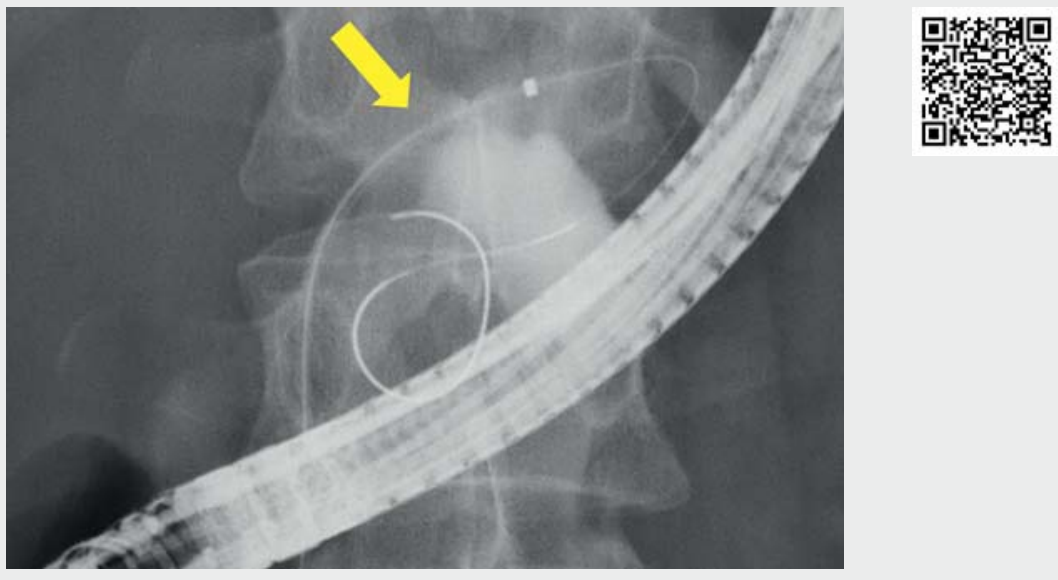

Video 1 Intentional endoscopic nasopancreatic drainage to a pancreatic fistula in the treatment of disconnected pancreatic duct syndrome.

firmed the absence of leakage by contrast pancreatography ( $\triangleright$ Fig.5) and removed the stent. The patient was cured and did not develop complications, including diabetes mellitus. The technique enables bridging of a completely disrupted MPD in patients with DPDS.

\section{Endoscopy_UCTN_Code_TTT_1AR_2AI}

\section{Competing interests}

The authors declare that they have no conflict of interest.

The authors

Yasuki Hori, Kazuki Hayashi, Itaru Naitoh, Michihiro Yoshida, Hiromi Kataoka

Department of Gastroenterology and

Metabolism, Nagoya City University Graduate

School of Medical Sciences, Nagoya, Japan

\section{Corresponding author}

\section{Yasuki Hori, MD, PhD}

Department of Gastroenterology and Metabolism, Nagoya City University Graduate School of Medical Sciences, 1 Kawasumi, Mizuho-cho, Mizuho-ku, Nagoya 467-8601, Japan

Fax: +81-52-8520952

yhori@med.nagoya-cu.ac.jp

\section{References}

[1] Varadarajulu S, Noone TC, Tutuian R et al. Predictors of outcome in pancreatic duct disruption managed by endoscopic transpapillary stent placement. Gastrointest Endosc 2005; 61: 568-575

[2] Hori Y, Vege SS, Chari ST et al. Classic chronic pancreatitis is associated with prior acute pancreatitis in only $50 \%$ of patients in a large single-institution study. Pancreatology 2019; 19: 224-229

[3] Lawrence C, Howell DA, Stefan AM et al. Disconnected pancreatic tail syndrome: potential for endoscopic therapy and results of long-term follow-up. Gastrointest Endosc 2008; 67: 673-679

[4] Pelaez-Luna M, Vege SS, Petersen BT et al. Disconnected pancreatic duct syndrome in severe acute pancreatitis: clinical and imaging characteristics and outcomes in a cohort of 31 cases. Gastrointest Endosc 2008; 68: 91-97

[5] Pearson EG, Scaife CL, Mulvihill S] et al. Roux-en-Y drainage of a pancreatic fistula for disconnected pancreatic duct syndrome after acute necrotizing pancreatitis. HPB (Oxford) 2012; 14: 26-31

\section{Bibliography}

Endoscopy 2021; 53: E295-E296

DOI 10.1055/a-1264-6949

ISSN 0013-726X

published online 8.10 .2020

(c) 2020. Thieme. All rights reserved.

Georg Thieme Verlag KG, Rüdigerstraße 14,

70469 Stuttgart, Germany 